\title{
Physical processes in the damping of electromechanical oscillations of the synchronous machine with magnitude-phase excitation controller
}

\author{
Oleg Agamalov \\ TPSPP, Yuzhnoukrainsk, Ukraine
}

Email address:

olegagamalov@gmail.com

\section{To cite this article:}

Oleg Agamalov. Physical Processes in the Damping of Electromechanical Oscillations of the Synchronous Machine with Magnitude-Phase Excitation Controller. International Journal of Energy and Power Engineering. Vol. 2, No. 4, 2013 , pp. $164-171$. doi: $10.11648 /$ j.ijepe.20130204.14

\begin{abstract}
Physical processes the damping of the synchronous machine electromechanical oscillations without excitation control, with proportional automatic voltage regulator (AVR-P), and magnitude-phase excitation controller (MPH-EC), which is responsive to the deviations of the magnitude and phase of the terminal voltage phasor are considered. The advantages of the MPH-EC, compared with the above other structures excitation control are discussed. A calculation example of the small signal stability gain margin at active power of the synchronous machine with the above structures of the excitation controllers for the simplest model "single machine - infinite bus" is examined. From this example follows, that the variable small signal stability gain margin of the MPH-EC exceeds that parameter for AVR-P, and excitation control with power system stabilizer (AVR+PSS) with a some adjustable rotor angle deviation.
\end{abstract}

Keywords: Synchronous Generator, Terminal Voltage Phasor, Automatic Voltage Regulator, Small Signal Stability Gain Margin, Control by Plant Complex Output

\section{Introduction}

Currently, in theory and practice of the excitation control of the synchronous machines, the fundamental role played by the feedback on the terminal voltage magnitude $[1,2]$. Excitation control is based on the calculation of the scalar mismatch error between the reference (set point) $V_{\text {ref }}$ and the measured terminal voltage $V_{t}$. The obtained scalar error $\Delta V=V_{\text {ref }}-V_{t}$ is used as the input of a PID controller or it simplified variants (PI, PD) [1-3] for controlling the excitation current $I_{f}$ of the synchronous machine. The purpose of this regulation is the precise maintaining the synchronous machine terminal voltage $V_{t}$ according to the setpoint $V_{r e f}$ in all possible operating conditions. For this necessary to set the greatest possible value of the proportional gain of the automatic voltage regulator (AVR). However, as has been determined theoretically, and observed in practice [4], in some operating conditions of the synchronous machines this leads to a decrease of the damping component $T_{D}$ of the electric moment, that is proportional to the rotor speed deviation $\Delta \omega$, and to the electromechanical oscillations in the event of grid disturbances. Therefore, additionally in excitation control of the synchronous machines are used the feedbacks on the parameters that characterizing the rotor motion: frequency deviation $\Delta f$, rotor speed deviation $\Delta \omega$, field current deviation $\Delta I_{f}$ or accelerating power $P_{a}$. These additional feedbacks are implemented as stabilizing channels in the automatic excitation regulator of strong action AER-SA in Russia [2], or as separate devices - power system stabilizers (PSS). This greatly complicates the design, subsequent coordination of regulation and stabilization channels, holding of the commissioning works, and operation of the excitation control.

Therefore represents of interest the development of excitation control, which implements a new type of the complex feedback [5-8]. The structure of that feedback both reflects the electromagnetic state, determined by terminal voltage, and electromechanical state, defined by the rotor movement of the synchronous machine. For this necessary to increase the dimension of the mismatch error in such a way that it reflects the deviations of the terminal voltage and rotor angle of the synchronous machine. Corresponds to this requirement, as input of the excitation control, used the 
terminal voltage phasor of the synchronous machine. Physical processes in the damping of electromechanical oscillations of the synchronous machine with magnitude-phase excitation controller (MPH-EC), using as input the terminal voltage phasor (complex output of the plant - the synchronous generator), are considered in this article with the following sections. The second section presents the main provisions, used in the MPH-EC development, and it is algorithm. The third section discusses the physical processes of electromechanical oscillations damping for the synchronous machine without excitation control, with proportional excitation controller AVR-P, and magnitude-phase excitation controller MPH-EC. A calculation example of the small signal stability gain margin at active power of the synchronous machine with the above structures of the excitation controllers for the simplest model "single machine - infinite bus" is given in the fourth section. As can be seen from the above example, MPH-EC have the variable small signal stability gain margin at active power, that exceeds the value of that parameter for AER-SA or AVR+PSS with a some adjustable rotor angle deviation. Conclusion summarizes the content of this article, and defines the future directions of the work at MPH-EC.

\section{Main Provisions and MPH-EC Algorithm}

We define the set point of the excitation controller as phasor $\bar{V}_{\text {ref }}$ (p. u.), which coincides in the steady-state with the field current $I_{f}$ (p. u.) or synchronous e.m.f. phasor $\bar{E}_{q}$ (p. u.). The magnitudes of the set point and terminal voltage phasors are equal $\left|\bar{V}_{r e f}\right|=\left|\bar{V}_{t 0}\right|$, (p. u.), but shifted by the steady-state rotor angle $\delta_{S M 0}$. Thus, it may be determined the normal complex argument error function NCAEF [5-8] of the excitation control for the steady state of the synchronous machine with the terminal voltage $\bar{V}_{t 0}=V_{q 0}+j V_{d 0}$, and the CAEF increment in the transient, in accordance with the vector diagram, Fig. 1:

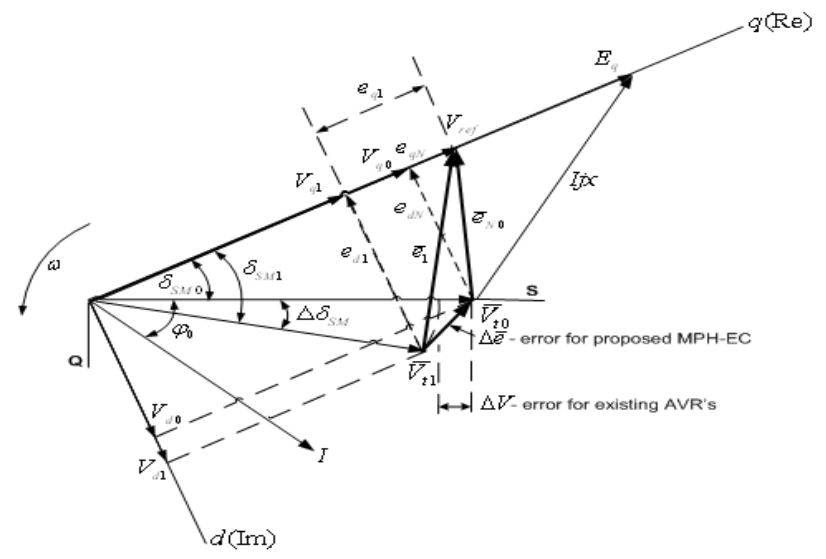

Figure 1. Vector diagram of synchronous machine, showing the CAEF increment $\Delta \bar{e}$ by the terminal voltage phasor $\overline{V_{t}}$.

$$
\begin{aligned}
& \bar{e}_{N 0}=f\left(\bar{V}_{r e f}, \bar{V}_{t 0}\right)= \\
& \bar{V}_{r e f}-\bar{V}_{t 0}=V_{r e f}-\left(V_{t 0} \cos \delta_{S M 0}+j V_{t 0} \sin \delta_{S M 0}\right)= \\
& =\left(V_{r e f}-V_{q 0}\right)-j V_{d 0}=e_{q N}+j e_{d N}
\end{aligned}
$$

where:

$$
\begin{aligned}
& e_{q N}=V_{r e f}-V_{t 0} \cos \delta_{S M 0}=V_{r e f}-V_{q 0}, \\
& e_{d N}=-V_{t 0} \sin \delta_{S M 0}=-V_{d 0} .
\end{aligned}
$$

NCAEF by terminal voltage phasor (1) characterized by the equation $\left|\bar{V}_{r e f}\right|=\left|\bar{V}_{t 0}\right|$, (p. u.), and defines the steady state of the inertial element - the rotor of the synchronous machine with the terminal voltage phasor angle $\delta_{S M 0}=$ const .

In case of violation the steady-state of the synchronous machine, as a result of a disturbance in the power system, and the corresponding terminal voltage phasor $\bar{V}_{t 1}$, the transient $\mathrm{CAEF} \quad \bar{e}_{1} \neq \bar{e}_{N 0}$ :

$$
\begin{aligned}
& \bar{e}_{1}=f\left(\bar{V}_{r e f}, \bar{V}_{t 1}\right)= \\
& =\bar{V}_{r e f}-\bar{V}_{t 1}=V_{r e f}-\left(V_{t 1} \cos \delta_{S M 1}+j V_{t 1} \sin \delta_{S M 1}\right)= \\
& =\left(V_{r e f}-V_{q 1}\right)-j V_{d 1}=e_{q}+j e_{d}
\end{aligned}
$$

which leads to CAEF increment $\Delta \bar{e}$ :

$$
\begin{aligned}
& \Delta \bar{e}=f\left(\Delta V_{t}, \Delta \delta_{S M}\right)=\bar{e}_{N 0}-\bar{e}_{1}= \\
& =\left(V_{t 1} \cos \delta_{S M 1}-V_{t 0} \cos \delta_{S M 0}\right)+ \\
& +j\left(V_{t 1} \sin \delta_{S M 1}-V_{t 0} \sin \delta_{S M 0}\right)= \\
& =\left(V_{q 1}-V_{q 0}\right)+j\left(V_{d 1}-V_{d 0}\right)
\end{aligned}
$$

The CAEF increment $\Delta \bar{e}$ (3), taking into account $\left|V_{t 0}\right|=\left|V_{r e f}\right|$, reflects the increments of the terminal voltage magnitude $\Delta V_{t}$ and rotor angles $\Delta \delta_{S M}$, and generalizes the traditional definition of the error by magnitude terminal voltage, that used in the existing excitation control:

$$
\begin{aligned}
& \Delta \bar{e}=\left|\bar{V}_{t 0}-\bar{V}_{t 1}\right|=\sqrt{V_{r e f}^{2}+V_{t 1}^{2}-2 V_{r e f} V_{t 1} \cos \left(\Delta \delta_{S M}\right)}= \\
& =\sqrt{\left(V_{q 1}-V_{q 0}\right)^{2}+\left(V_{d 1}-V_{d 0}\right)^{2}} \\
& \Delta V_{t}=V_{r e f}-V_{t 1}=V_{t 0}-V_{t 1}=\sqrt{V_{q 0}^{2}+V_{d 0}^{2}}-\sqrt{V_{q 1}^{2}+V_{d 1}^{2}}
\end{aligned}
$$

For excitation control of the synchronous machine the CAEF increment should be regarded as a real function of a complex argument - the terminal voltage phasor that changes in transient:

$$
\frac{d(\Delta \bar{e})}{d t}=\frac{\partial\left(\Delta e\left(\bar{V}_{t}\right)\right)}{\partial \bar{V}_{t}} \frac{d \bar{V}_{t}}{d t}
$$

where $\frac{\partial\left(\Delta e\left(\bar{V}_{t}\right)\right)}{\partial \bar{V}_{t}}$ - the gradient of the CAEF increment by 
the terminal voltage phasor, $\frac{d \bar{V}_{t}}{d t}=\dot{\bar{V}}_{t}$ - a derivative of the terminal voltage phasor. Given the vector diagram, Fig. 1, we define the terminal voltage phasor and CAEF increment in transient, in coordinate system $S-Q$ (for the initial steady state):

$$
\begin{aligned}
& \left|\bar{V}_{t 0}\right|=\left|\bar{V}_{r e f}\right| \\
& \bar{V}_{t 1}=V_{t s}+j V_{t q}=V_{t 1} \cos \left(\Delta \delta_{S M}\right)+j V_{t 1} \sin \left(\Delta \delta_{S M}\right)= \\
& =V_{t 0} e^{j\left(\Delta \delta_{S M}\right)} \\
& \widetilde{V}_{t 1}=V_{t s}-j V_{t q}=V_{t 0} \cos \left(\Delta \delta_{S M}\right)-j V_{t 0} \sin \left(\Delta \delta_{S M}\right)= \\
& =V_{t 0} e^{j\left(-\Delta \delta_{S M}\right)} \\
& V_{t s}=V_{t 1} \cos \left(\Delta \delta_{S M}\right), V_{t q}=V_{t 1} \sin \left(\Delta \delta_{S M}\right) \\
& \Delta \bar{V}_{t}=\Delta V_{t s}+j\left(\Delta V_{t q}\right)= \\
& =V_{t 1} \cos \left(\Delta \delta_{S M}\right)-V_{t 0}+j V_{t 1} \sin \left(\Delta \delta_{S M}\right)= \\
& =V_{t 1} \cos \left(\Delta \delta_{S M}\right)-V_{r e f}+j V_{t 1} \sin \left(\Delta \delta_{S M}\right) \\
& \Delta \widetilde{V}_{t}=\Delta V_{t s}-j\left(\Delta V_{t q}\right)= \\
& =V_{t 1} \cos \left(\Delta \delta_{S M}\right)-V_{t 0}-j V_{t 1} \sin \left(\Delta \delta_{S M}\right)= \\
& =V_{t 1} \cos \left(\Delta \delta_{S M}\right)-V_{r e f}-j V_{t 1} \sin \left(\Delta \delta_{S M}\right), \\
& \Delta V_{t s}=V_{t 1} \cos \left(\Delta \delta_{S M}\right)-V_{t 0}= \\
& =V_{t 1} \cos \left(\Delta \delta_{S M}\right)-V_{r e f}, \Delta V_{t q}=V_{t 1} \sin \left(\Delta \delta_{S M}\right) \\
& \Delta \bar{e}=-\Delta \bar{V}_{t}=-\left(\Delta V_{t s}+j\left(\Delta V_{t q}\right)\right)= \\
& =-\left(V_{t 1} \cos \left(\Delta \delta_{S M}\right)-V_{t 0}+j V_{t 1} \sin \left(\Delta \delta_{S M}\right)\right)= \\
& =-\left(V_{t 1} \cos \left(\Delta \delta_{S M}\right)-V_{r e f}+j V_{t 1} \sin \left(\Delta \delta_{S M}\right)\right)= \\
& =V_{r e f}-V_{t 1} \cos \left(\Delta \delta_{S M}\right)-j V_{t 1} \sin \left(\Delta \delta_{S M}\right)= \\
& =\Delta e_{S}+j\left(\Delta e_{q}\right), \\
& \Delta e_{S}=V_{r e f}-V_{t 1} \cos \left(\Delta \delta_{S M}\right), \Delta e_{q}=-V_{t 1} \sin \left(\Delta \delta_{S M}\right)
\end{aligned}
$$

Non-constant real-valued function of a complex variable(s) is non-analytic, and therefore not differentiable in the accepted sense (Cauchy-Riemann condition). The calculation of the CAEF increment based on the Wirtinger partial derivatives [5-8]. Oriented an output of excitation control in the direction of the antigradient (5) we obtain the following algorithm for MPH-EC:

$$
\begin{aligned}
& u=K\left(V_{r e f}-\frac{2 \cos \left(N \Delta \delta_{S M}\right)}{1+\cos \left(2 N \Delta \delta_{S M}\right)} V_{t}\right)= \\
& =K\left(V_{r e f}-V_{t} \sec \left(N \Delta \delta_{S M}\right)\right)
\end{aligned}
$$

where $K$ - gain of the proportional channel by the magnitude deviation of the terminal voltage phasor $\left|\Delta V_{t}\right|$, and $N$ - gain by rotor angle deviation $\Delta \delta_{S M}$. A more detailed derivation of the magnitude-phase control algorithm is given in [5-9].

\section{Physical Processes in the Damping of Electromechanical Oscillations of the Synchronous Machine with Different Structures of the Excitation Control}

\subsection{Non-Regulated Synchronous Machine}

As it is known, that for non-regulated synchronous machine, the main factor that mitigated it electromechanical oscillations is their damping or asynchronous moment. So, in transient the full electric power of the synchronous machine is given as $[1,10]$ :

$$
\begin{aligned}
& P_{e}=P_{e S}+P_{e D}= \\
& =\frac{E_{q}^{\prime} V_{b}}{x_{\Sigma}^{\prime}} \sin \delta-\frac{V_{b}^{2}\left(x_{\Sigma}-x_{\Sigma}^{\prime}\right)}{2 x_{\Sigma} x_{\Sigma}^{\prime}} \sin 2 \delta+K_{D} \frac{d(\Delta \delta)}{d t}
\end{aligned}
$$

where: $P_{e}, P_{e S}, P_{e D}$ - electric power, and it synchronous and damping components, $E_{q}^{\prime}$ - transient synchronous e.m.f., $V_{b}$ - bus voltage, $x_{\Sigma}^{\prime}$ - total transient inductance, $\delta$ rotor angle, $x_{\Sigma}$ - total tie inductance, $K_{D}$ - damper gain. On the assumption, that the turbine power is constant $P_{t}=$ const , the rotor angle deviation is determined based on the 2nd Newton's law as:

$$
\begin{aligned}
& T_{j} \frac{d^{2}(\Delta \delta)}{d t^{2}}=P_{t}-P_{e}=P_{t}-\left(P_{e S}+P_{e D}\right)= \\
& P_{t}-\left(\frac{E_{q}^{\prime} V_{b}}{x_{\Sigma}^{\prime}} \sin \delta-\frac{V_{b}^{2}\left(x_{\Sigma}-x_{\Sigma}^{\prime}\right)}{2 x_{\Sigma} x_{\Sigma}^{\prime}} \sin 2 \delta+K_{D} \frac{d(\Delta \delta)}{d t}\right)
\end{aligned}
$$

where $T_{j}$ - the mechanical inertia constant of the unit. Then:

$$
\begin{aligned}
& T_{j} \frac{d^{2}(\Delta \delta)}{d t^{2}}+K_{D} \frac{d(\Delta \delta)}{d t}+ \\
& +\left(\frac{E_{q}^{\prime} V_{b}}{x_{\Sigma}^{\prime}} \cos \delta_{0}-\frac{V_{b}^{2}\left(x_{\Sigma}-x_{\Sigma}^{\prime}\right)}{x_{\Sigma} x_{\Sigma}^{\prime}} \cos 2 \delta_{0}\right) \cdot \Delta \delta=0
\end{aligned}
$$

with the characteristic equation:

$$
\begin{aligned}
& T_{j} p^{2}+K_{D} p+ \\
& +\left(\frac{E_{q}^{\prime} V_{b}}{x_{\Sigma}^{\prime}} \cos \delta_{0}-\frac{V_{b}^{2}\left(x_{\Sigma}-x_{\Sigma}^{\prime}\right)}{x_{\Sigma} x_{\Sigma}^{\prime}} \cos 2 \delta_{0}\right)=0
\end{aligned}
$$

and it roots:

$$
p_{1,2}=\frac{-K_{D} \pm \sqrt{K_{D}^{2}-4 T_{j}\left(\frac{E_{q}^{\prime} V_{b}}{x_{\Sigma}^{\prime}} \cos \delta_{0}-\frac{V_{b}^{2}\left(x_{\Sigma}-x_{\Sigma}^{\prime}\right)}{x_{\Sigma} x_{\Sigma}^{\prime}} \cos 2 \delta_{0}\right)}}{2 T_{j}}
$$

that defines the rotor motion. After reaching the some deviation $\Delta \delta$, arising due to the acceleration power $\Delta P=P_{e}-P_{t}$, the rotor angle deviation begins to decrease, 
i.e. $d(\Delta \delta) / d t<0$. Therefore on the section $A_{1} A_{2}$ of the angular characteristic $P_{e D}<0$, and the acceleration power will decline more rapidly than under the action only the synchronizing component $P_{e S}$, Fig. 2 [10]:

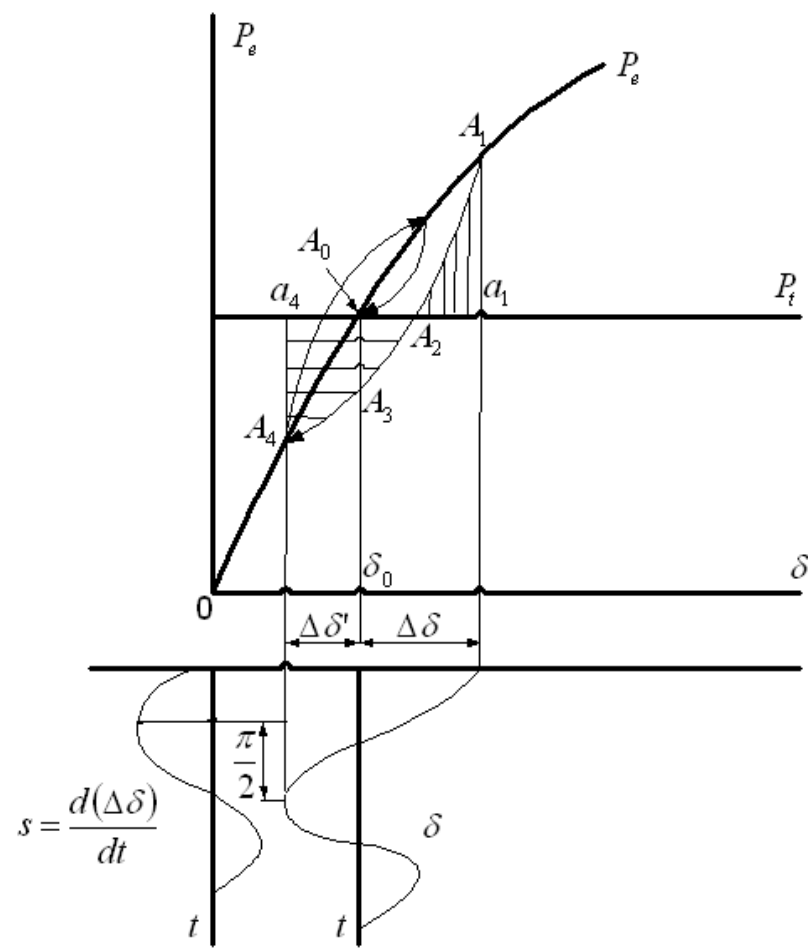

Figure 2. Damping of the electromechanical oscillation for non-regulated synchronous machine.

Braking power imbalance reduces the kinetic energy of the rotor on the section $A_{1} A_{2}$. With use the areas method, the decrease of the kinetic energy is determined as proportional to the area $A_{1} A_{2} a_{1}$. At the point $A_{2}$, where $P_{e}=P_{t}$, due to the reduction of the rotor kinetic energy on the section $A_{1} A_{2}$, the rotor speed is $\omega_{A 2}<\omega_{0}$. Therefore, further the rotor angle decreasing relative to the initial value as $\Delta \delta^{\prime}$. At the section $A_{2} A_{3} A_{4}$ we have $P_{e}<P_{t}$, and the rotor begins to accelerate. In the point $A_{4}$ the rotor speed $\omega_{A 4}=\omega_{0}$ that leads to the subsequent rise of the rotor angle, as shown in Fig. 2. So, as indicated in [10], the dynamic angular characteristic of the synchronous machine in transient at section $A_{1} A_{4}$ is below than for it static angular characteristics due to the damping torque $P_{e D}=K_{D}(d(-\Delta \delta) / d t)$. Accordingly, when the rotor angle returns to the initial value $\delta_{0}$, the electric power is $P_{e}<P_{t}$ by the value $P_{e D}$. This leads to an earlier acceleration of the rotor in the point $A_{2}$, and equalization of it kinetic energy deviations during oscillations (i.e. the equality of the acceleration and deceleration areas) already at the smaller value the rotor angle deviation $\Delta \delta^{\prime}<\Delta \delta$. As a result of the subsequent oscillations $\Delta \delta \rightarrow 0$ in the synchronous machine with the damper windings.

\subsection{Synchronous Machine with AVR-P}

Next, consider the physical process of the electromechanical oscillations in the regulated synchronous machine with the proportional AVR (AVR-P). It is known [1, $2,10]$, the positive effect of the proportional excitation control of the synchronous machine consists in the increasing the synchronizing component of the electrical power due to the increase of their synchronous e.m.f. $E_{q} \approx I_{f}$ at transients. At the same time, as noted in references $[1,2,10]$ the fast-acting proportional excitation control due to the great feedback gain $K_{0 V}>50$ in accordance with the law:

$$
V_{f}=V_{f 0}+K_{0 V}\left(V_{\text {ref }}-V_{t}\right)
$$

can lead to the oscillatory instability of a synchronous machine, Fig. 3:

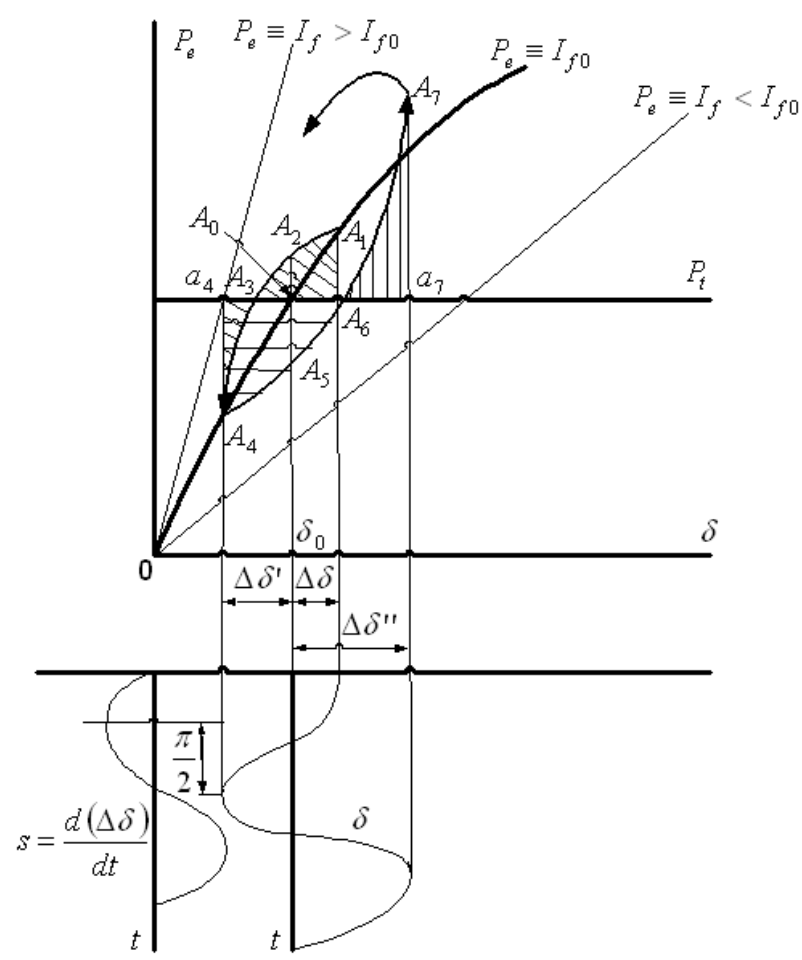

Figure 3. Damping of the electromechanical oscillation for synchronous machine with AVR-P.

After first swing $\Delta \delta$, the rotor angle return to the steady state value $\delta_{0}$ on the section $A_{1} A_{2}$. AVR-P reacting to the terminal voltage drop, increases the field current $I_{f} \sim E_{q}$. Herewith, the acceleration power is $\Delta P=P_{e}-P_{t} \neq 0$ (point $A_{2}$ ). Power equality will be achieved at the point $A_{3}$, and the equality of the acceleration and deceleration areas at the point $A_{4}$. As a result of this delay and powers disparities for the rotor angle $\delta_{0}$ in point $A_{2}$, the rotor angle deviation at point $A_{4}$ will be greater than the initial deviation $\Delta \delta^{\prime}>\Delta \delta$. Due to the rotor angle deviation $\Delta \delta^{\prime}$, and the corresponding it the terminal voltage increases, AVR-P will 
reduce the field current $I_{f}<I_{f 0}$. Therefore, the subsequent increase in the rotor angle to the initial steady-state value $\delta=\delta_{0}$ corresponds to larger accelerating power at point $A_{5}$. The powers equality $P_{e}=P_{t}$ will be achieved only at a point $A_{6}$. Therefore, the acceleration area will be equalized at the larger rotor angle deviation $\Delta \delta^{\prime \prime}>\Delta \delta^{\prime}>\Delta \delta$ at point $A_{7}$. As a result, the moving point of the angular characteristics $P_{e}=f(\delta)$ describes the unwinding spiral, which determines the unstable oscillatory mode of the synchronous machine. The resulting damping moment depends on the initial operation mode and parameters of the synchronous machine and grid. With small initial steady state rotor angle $\delta_{0}$, the synchronization component $\partial P_{e} / \partial \delta$ of the electrical power is significant, which leads to higher values of the rotor speed deviation (slip) at the electromechanical oscillations, and thus a large positive damping component of the electrical power. With a large initial steady state rotor angle $\delta_{0}$, the synchronization component $\partial P_{e} / \partial \delta$ of the electrical power is negligible, so the amplitude of the electro-mechanical oscillations is small, and therefore is small the positive damping component of the electric power, i.e. the probability of the oscillatory instability is higher. To suppress the oscillatory instability currently used the structure of the excitation control with AVR and power system stabilizer (PSS) in which additionally implemented feedbacks by parameters of the synchronous machine electromechanical state (rotor speed, frequency, accelerating power). Additional feedbacks are needed for biasing the phase lag between the field current $I_{f}(t)$ (e.m.f. $\left.E_{q}(t)\right)$ and the rotor angle $\delta(t)$ for positive damping moment of the excitation control, i.e. so that with a decrease in the rotor angle to $\delta(t)=\delta_{0}$ at the first swing of the electromechanical oscillations is provided the condition $P_{e}<P_{t}$.

\subsection{Synchronous Machine with MPH-EC}

For the qualitative analysis we neglect the moment at damping windings of synchronous machine, which is always positive $P_{e D}>0$, and assume that the field current $I_{f}$ is determined only by the excitation control, i.e. the voltage, applied to the field winding $V_{f}$. The excitation control law with MPH-EC is:

$$
\begin{aligned}
& V_{f}=V_{f 0}+K_{\Sigma}\left(V_{r e f}-\frac{2 \cos (N \Delta \delta)}{1+\cos (2 N \Delta \delta)} V_{t}\right)= \\
& =V_{f 0}+K_{\Sigma}\left(V_{r e f}-V_{t} \sec (N \Delta \delta)\right)
\end{aligned}
$$

In accordance with (14), the measured terminal voltage deviation $\Delta V_{t}$ of synchronous machine additionally corrected as a function of the rotor angle deviation $\sec \left(N \Delta \delta_{S M}\right)$, where $N$ - the gain by $\Delta \delta$. At the same setpoint $\Delta V_{\text {ref }}=0$ equation (14) in the increments is:

$$
\Delta V_{f}=K_{\Sigma} \frac{2 \cos (N \Delta \delta)}{1+\cos (2 N \Delta \delta)} \Delta V_{t}=K_{\Sigma} \sec (N \Delta \delta) \Delta V_{t}
$$

and it is possible to determine the feedback gain of the excitation control by the terminal voltage phasor as follows:

$$
K_{F}=K_{\Sigma} \frac{2 \cos (N \Delta \delta)}{1+\cos (2 N \Delta \delta)}=K_{\Sigma} \sec (N \Delta \delta)
$$

The magnitude and sign of the gain $K_{F}$ depends on the argument $(N \Delta \delta)$. If:

$$
(N \Delta \delta)>\pi / 2 \Rightarrow \Delta \delta_{L}>\pi / 2 N
$$

then changes the sign of $K_{F}$, according to the function graph $\sec \left(N \Delta \delta_{S M}\right)$, Fig. 4:

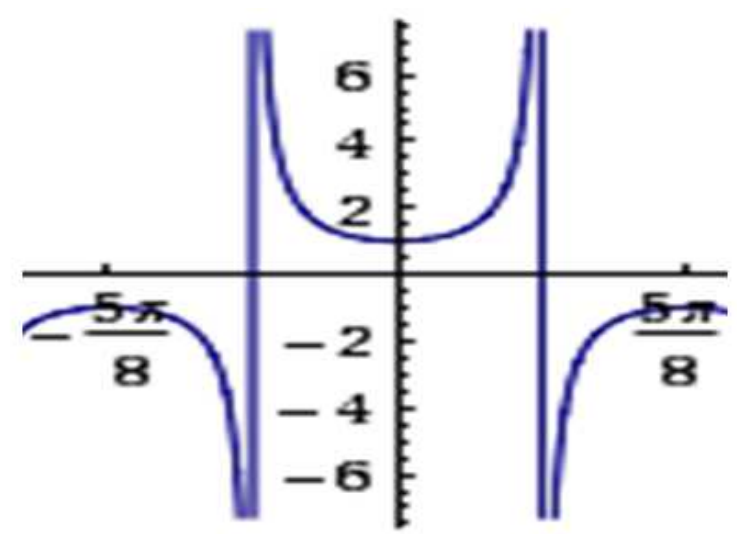

$$
N=1.6\left(\Delta \delta_{L}=56^{\circ}\right)
$$

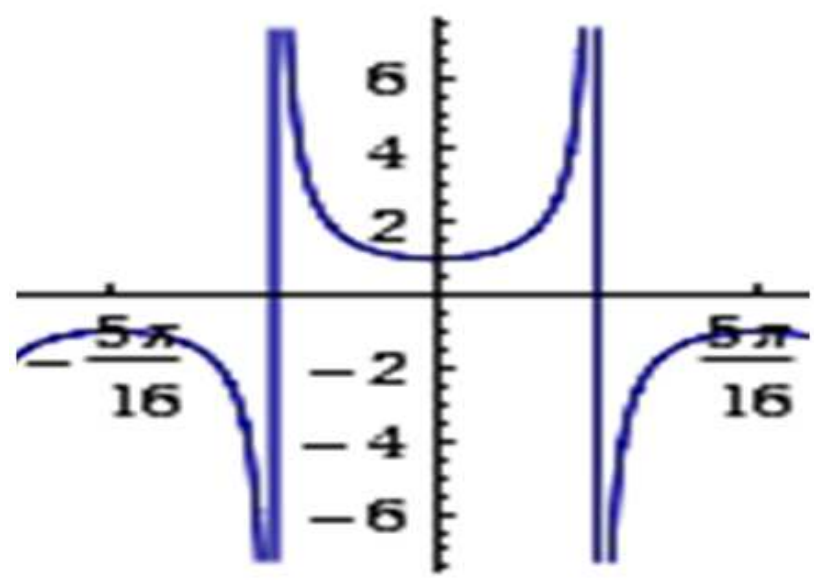

$$
N=3.2\left(\Delta \delta_{L}=28^{\circ}\right)
$$

Figure 3. Graph of the function $2 \cos (N \Delta \delta) /(1+\cos (2 N \delta))=\sec (N \Delta \delta)$.

Equations $(15,16)$ with inequality (18) are defines a significant positive impact of MPH-EC on the synchronizing component of the electrical power due to non-linear increase of the synchronous e.m.f.

$\Delta E_{q} \approx \Delta I_{f} \approx \Delta V_{f}=K_{\Sigma} \sec (N \Delta \delta) \Delta V_{t} \quad$ in transients at $\Delta \delta<\Delta \delta_{L}$. It should be noted the "concavity" of characteristics, shown in Fig. 4. That corresponds to a 
slight increase of the gain $K_{F}$ at a small rotor angle deviation (for example, with a planned increase in the synchronous machine power), and its intense increase at significant rotor angle deviation up to $\Delta \delta_{L}$.

Now consider the impact of the MPH-EC on the synchronous machine stability, Fig. 5:

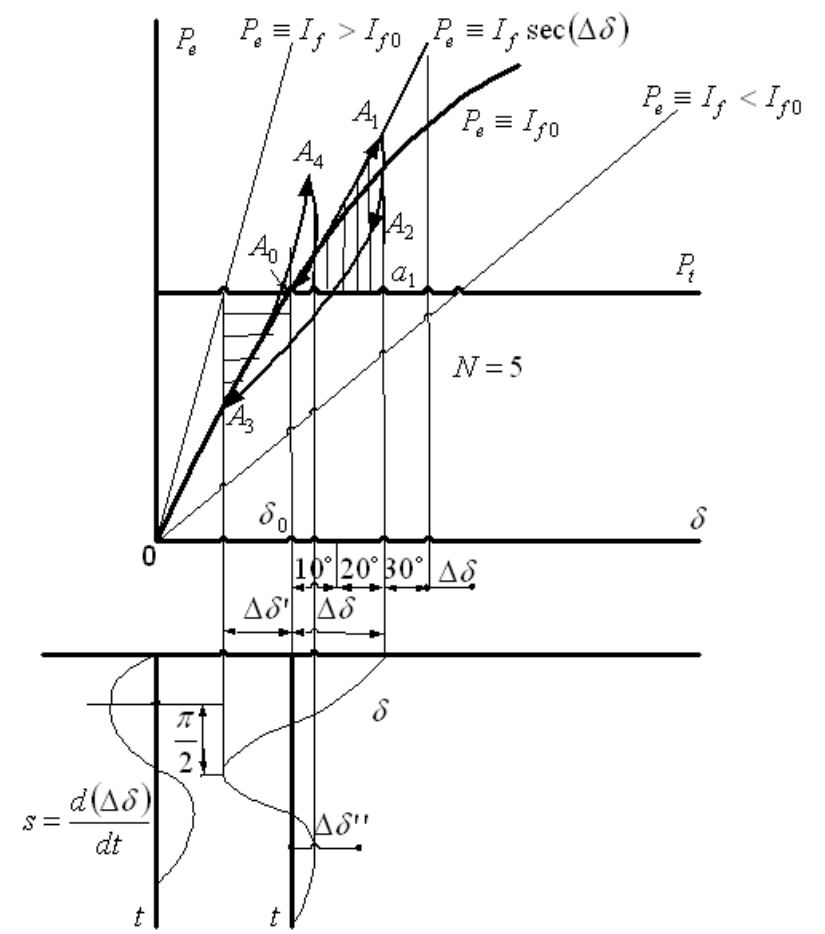

Figure 4. Damping of the electromechanical oscillations for synchronous machine with MPH-EC.

Suppose, that as a result of the disturbance in the power system, the rotor angle deviation occurs $\Delta \delta \rightarrow \Delta \delta_{L}$, where the value $\Delta \delta_{L}=18^{\circ}$ el. on the Fig. 5 , defined for $N=5$. The excitation control equations $(14,15)$ are determining an increase of the static angular characteristics in 1.05 times for section $A_{0} A_{1}$ according to a predetermined value $N=5$. At $\Delta \delta_{L}=18^{\circ}$ el., in accordance with $(16,17)$, the sign of the gain $K_{F}$ changes. This leads to the de-excitation of the synchronous machine, and the transition to point $A_{2}$. Arisen braking power imbalance leads to a reduction of the rotor kinetic energy on the section $A_{2} A_{3}$ with a simultaneous increase of the e.m.f. $\Delta E_{q} \approx \Delta I_{f} \approx \Delta V_{f}=K_{\Sigma} \Delta V_{t}$, as a result of the terminal voltage deviation relative to the setpoint, and decreasing of the rotor angle deviation $\Delta \delta<\Delta \delta_{L}$. The equality of acceleration and deceleration areas will be achieved at a point $A_{3}$ at a smaller deviation $\Delta \delta^{\prime}$. With further increase in the rotor angle $\Delta \delta^{\prime \prime}$ on the section $A_{3} A_{4}$ under the accelerating power imbalance, for point $A_{4}$ corresponds to less braking power imbalance due to the previous de-excitation action of MPH-EC, depending on the rotor angle deviation $\Delta \delta^{\prime}$, i.e. $\Delta \delta^{\prime \prime}<\Delta \delta^{\prime}<\Delta \delta$. Thus, MPH-EC provides such phase relation between the deviations of e.m.f. $E_{q}^{\prime}$ (field current $\Delta I_{f}$ ), and the rotor angle $\Delta \delta$ of the synchronous machine, that would as the rotor angle move towards to the initial value "from above" $\delta_{+} \rightarrow \delta_{0}$, the electric power of the synchronous machine was less than the corresponding turbine power $P_{e i}<P_{t i}$ for the angle $\delta_{0}<\delta_{i}<\delta_{+}$. Conversely, with an increase the rotor angle to the initial value "from bottom" $\delta_{-} \rightarrow \delta_{0}$, the electrical power of the synchronous machine is greater $P_{e j}>P_{t j}$ for rotor angle $\delta_{-}<\delta_{j}<\delta_{0}$. As a result, on the angular characteristics $P_{e}=f(\delta)$ the moving point describes a convergent curve that defines the decaying oscillation mode of the synchronous machine.

\section{The Calculation of the Small Signal Stability Gain Margin at Active Power of the Synchronous Machine with Different Structures of the Excitation Control}

Consider the impact of different excitation control structures on small signal stability gain margin at active power for the model "single machine - infinite bus" with the parameters in p. u.: $x_{d}=1.9 ; x_{T}=0.1 ; x_{L}=0.17$; $U_{10}=1.0 ; P_{10}=0.85 ; Q_{10}=0.264 ; x_{d}^{\prime}=0.38$.

4.1. For non-regulated synchronous machine the transmission power limit is determined thru e.m.f.:

$$
\begin{aligned}
& \dot{E}_{q 0}=\dot{U}_{10}+\frac{Q_{10}\left(x_{d}+x_{T}\right)}{U_{10}}+j \frac{P_{10}\left(x_{d}+x_{T}\right)}{U_{10}}= \\
& =1.528+j 1.7=2.286 e^{j 48.05^{\circ}}
\end{aligned}
$$

Busbar voltage the receiving system:

$\dot{U}_{0}=U_{10}-\frac{Q_{10} \cdot x_{o}}{U_{10}}-j \frac{P_{10} \cdot x_{o}}{U_{10}}=$

$0.955-j 0.145=0.966 e^{-j 8.633^{\circ}}$

Full rotor angle of the synchronous machine: $\delta_{0}=48.05^{\circ}+8.633^{\circ}=56.683^{\circ}$. The angular power characteristic:

$P_{0}=\frac{2,286 \cdot 0,966}{2,0+0,17} \sin 56.683^{\circ}=0.85$

which coincides with a predetermined active power of a synchronous machine. The transmission power limit at $\delta_{0}=90^{\circ}$ :

$P_{L}=\frac{E_{q 0} U_{0}}{x_{d \Sigma}}=\frac{2.286 \cdot 0.966}{2.17}=1.018$.

Thus, the small signal stability gain margin of the unregulated synchronous machine: 


$$
K_{S M}=\frac{1.018-0.85}{0.85} \cdot 100 \%=19.77 \% .
$$

4. 2. For excitation control with AVR-P the transmission power limit is determined at constant transient e.m.f. $E_{q}^{\prime}=$ const $[1,2]$, where:

$$
\begin{aligned}
& E_{q}^{\prime}=U_{10}+\frac{Q_{10}\left(x_{d}^{\prime}+x_{T}\right)}{U_{10}}+j \frac{P_{10}\left(x_{d}^{\prime}+x_{T}\right)}{U_{10}}= \\
& =1.127+j 0.408=1.2 e^{j 19.9^{\circ}}
\end{aligned}
$$

The full rotor angle is: $\delta_{0}^{\prime}=19,9+8,635=28,533^{\circ}$. In the steady state:

$P_{0}=\frac{E_{0}^{\prime} U_{0}}{x_{d \Sigma}} \sin \delta_{0}^{\prime}=\frac{1.2 \cdot 0.966}{0.38+0.1+0.17} \sin 28.533^{\circ}=0.85$

The transmission power limit:

$$
P_{L}^{\prime}=\frac{E_{0}^{\prime} U_{0}}{x_{d \Sigma}^{\prime}}=\frac{1.2 \cdot 0.966}{0.65}=1.783
$$

which corresponds to a small signal stability gain margin:

$$
K_{\text {AVR-P }}=\frac{1.783-0.85}{0.85} \cdot 100 \%=109.7 \% \text {. }
$$

4. 3. For excitation control with AVR+PSS, at the highest possible gain by terminal voltage deviation, we can define the small signal stability limit by taking $U_{S M}=$ const $[1,2]$ :

$$
\begin{aligned}
& U_{S M}=U_{10}+\frac{Q_{10} x_{T}}{U_{10}}+j \frac{P_{10} x_{T}}{U_{10}}= \\
& =1.026+j 0.085=1.029 e^{j 4.736^{\circ}}
\end{aligned}
$$

The full rotor angle in this case

$$
\delta_{U_{S M}}=4.736^{\circ}+8.633^{\circ}=13.369^{\circ}
$$

In the steady state:

$$
P_{0}=\frac{U_{S M} U_{0}}{x_{T}+x_{o}} \sin \delta_{U_{S M}}=\frac{1.029 \cdot 0.966}{0.1+0.17} \sin 13.369^{\circ}=0.85
$$

The transmission power limit:

$$
P^{\prime \prime}{ }_{L}=\frac{U_{S M} U_{0}}{x_{T}+x_{L}}=\frac{1.029 \cdot 0.966}{0.27}=3.68
$$

which corresponds to a small signal stability gain margin:

$$
K_{A V R+P S S}=\frac{3.68-0.85}{0.85} 100 \%=333 \% .
$$

4. 4 For excitation control with MPH-EC $(14,15)$ the transmission power limit we define, based on the dependence of the gain $K_{F}$, and therefore, the transient e.m.f. as a function of the rotor angle deviation
$E_{q}^{\prime}=f(\sec (N \Delta \delta))$. Then, for different variations of the rotor angle, and the previously defined system parameters, we can calculate the variable transmission power limit as:

$$
P_{M F}^{\prime}=\frac{E_{0}^{\prime} U_{0}}{x_{d \Sigma}^{\prime}} \sec (N \Delta \delta)=\frac{1.2 \cdot 0.966}{0.65} \sec (N \Delta \delta)=1.783 \sec (N \Delta \delta)
$$

and variable small signal stability gain margin:

$$
K_{M F}=\frac{1.783 \sec (N \Delta \delta)-0.85}{0.85} \cdot 100 \%
$$

The obtained values of the variable small signal stability gain margin by the active power for a considered example with $N=5$ shown in the table 1 :

Table 1. The values of the variable small signal stability gain margin by the active power for synchronous machine with MPH-EC.

\begin{tabular}{lllllllll}
\hline$\Delta \delta,{ }^{\circ}$ el & $\mathbf{2}$ & $\mathbf{4}$ & $\mathbf{6}$ & $\mathbf{8}$ & $\mathbf{1 0}$ & $\mathbf{1 2}$ & $\mathbf{1 4}$ & $\mathbf{1 6}$ \\
\hline$K_{M F}, \%$ & 113 & 123 & 142 & 174 & 226 & 319 & 513 & 1107 \\
\hline
\end{tabular}

Comparing the results of the calculations, we see that an excitation control with AVR-P, compared with the case of the unregulated synchronous machine, increases the small signal stability gain margin by $90 \%$, and an excitation control with AVR+PSS by $223 \%$. Excitation control with MPH-EC is characterized by variable small signal stability gain margin by the active power that intensity increases with rotor angle deviations, and for $\Delta \delta>14^{\circ}$ el. is greater than for AVR+PSS. Increased of the transmission power limit, and small signal stability gain margin is caused by that AVR+PSS wholly or AVR-P partly eliminates the influence of synchronous machine impedance on it. MPH-EC, as previously discussed in section 3 , in the transient creates the effect of the negative differential resistance of the synchronous machine, reducing the voltage drop across with an increasing the stator current (power), and the rotor angle deviation. Thus achieving a variable small signal stability gain margin, exceeding the value for AVR+PSS with some adjustable value $\Delta \delta_{S M}$.

\section{Conclusions}

Algorithm and physical processes of damping the electromechanical oscillations of the synchronous machine for an excitation control with MPH-EC with complex input signal: the terminal voltage phasor, are considered. Parameters of given signal are used for regulating the terminal voltage magnitude, and for damping of electromechanical oscillations of the synchronous machine. That improves the transient and small-signal stability, minimizes the influence of the electromagnetic noise, improves reliability, and reduces the operating cost of excitation control as a result of the minimum set of the input signals, that are used (only phasors of the setpoint and terminal voltage). 
In order to determine the effectiveness of the proposed excitation control system structure in practice is necessary to examine its robust properties, by setting the structured and unstructured uncertainties in the models of the excitation system of the synchronous generator, and the power grid, which is the next step of the work.

\section{References}

[1] Kundur P. Power System Stability and Control / P. Kundur. New York: McGraw-Hill, 1994. - 1176 p.

[2] Yurganov A. A. Excitation Control of Synchronous Generators / A. A. Yurganov, V. A. Kogevnikov - Science, 1996. - 138 p. (in Russian).

[3] IEEE Std3.421.5-2005. IEEE Recommended Practice for Excitation System Models for Power System Stability Studies, 2005. $-85 \mathrm{p}$.

[4] Analysis and Control of Power System Oscillations: CIGRE TF 38.01.07, Brochure 111, 1996. - 119 p.

[5] Agamalov O. N. Excitation Control of Synchronous Machines using the Function of a Complex Error / O. N. Agamalov // XII International Conference "Problems of modern electrical engineering - 2012." - Vinnica, 2012. -
Special issue of the "Technical Electrodynamics» № 3. - p. 97-99. (in Russian).

[6] Agamalov O. N. AVR/PSS Structure by Terminal Voltage Phasor / O. N. Agamalov // AASRI Procedia Published by Elsevier B. V. selection and/or peer review under responsibility of American Applied Science Research Institute. - 2012. - Vol. 2. - p. 2-7.

[7] Agamalov O. N. Feedback of Control System by Deviations of Magnitude and Phase Delay of Plant Output / O. N. Agamalov // 8th WSEAS International Conference on Dynamical Systems and Control (CONTROL'12), Porto, Portugal, 2012. - p. 76-82.

[8] Agamalov O. N. Magnitude-Phase Controller (MPHC) / O. N. Agamalov // The 2012 International Conference on Control Engineering and Communication Technology (ICCECT 2012), Shenyang, China, 2012.

[9] Agamalov O. Control systems structures of synchronous machines excitation / O. Agamalov. - Lambert Academic Publishing, 2013. - 177 p. ISBN: 978-3-659-34203-5.

[10] Gurevich Y. E. Calculations of Stability and Emergency Control in Power Systems / Y. E. Gurevich, L. E. Libova, A. A. Okyn. - Energoatomizdat, 1990. - 390 p. (in Russian). 